\title{
Inhibited Spontaneous Emission by a Rydberg Atom
}

\author{
Randall G. Hulet, ${ }^{(a)}$ Eric S. Hilfer, and Daniel Kleppner \\ Research Laboratory of Electronics and Department of Physics, Massachusetts Institute of Technology, \\ Cambridge, Massachusetts 02139 \\ (Received 29 July 1985)
}

\begin{abstract}
Spontaneous radiation by an atom in a Rydberg state has been inhibited by use of parallel conducting planes to eliminate the vacuum modes at the transition frequency. Spontaneous emission is observed to "turn off" abruptly at the cutoff frequency of the waveguidelike structure and the natural lifetime is measured to increase by a factor of at least 20.
\end{abstract}

PACS numbers: $32.80 .-\mathrm{t}, 31.60 .+\mathrm{b}$

Spontaneous emission is often regarded as an unavoidable consequence of the coupling between matter and space. However, as one of the authors has pointed out, ${ }^{1,2}$ by surrounding the atom with a cavity which has no modes at the transition frequency, spontaneous emission can be inhibited or "turned off." Drexhage, in studies of fluorescence by dye molecules deposited on a dielectric film over a conducting plane, observed a decrease of up to $25 \%$ in the fluorescent decay rate due to cavitylike effects. ${ }^{3}$ Rydberg atoms provide the opportunity to achieve inhibited emission in microwave cavities. ${ }^{2}$ Vaidyanathan, Spencer, and Kleppner ${ }^{4}$ have reported a closely related effect-the suppression of blackbody absorption in Rydberg atoms by a parallel-plate cavity-and Gabrielse and Dehmelt ${ }^{5}$ have recently observed inhibited spontaneous emission of cyclotron radiation by an electron confined in a Penning trap. They observed damping times as much as 5 times longer than the free-space value and attributed the increased lifetime to cavity effects from the surrounding electrodes. Their experiment provided convincing evidence that spontaneous emission can be suppressed, but because of the difficulty of calculating the mode structure in the trap and varying it systematically, the demonstration was qualitative. In this Letter we describe the observation of inhibited spontaneous emission of a free atom by a well-characterized cavity whose properties can be varied systematically. Spontaneous emission by a Rydberg atom was observed to "switch off" abruptly as the transition wavelength was varied across the cavity's cutoff wavelength and the lifetime was measured to increase by a factor of at least 20.

The experiment employs Rydberg atoms in a "circular" state. ${ }^{6}$ This is a single-electron atomic state with large principal quantum number $n$, and magnetic quantum number $|m|=n-1$. The circular state is essential for this experiment because it radiates by only a single dipole transition. States with lower values of $|m|$ have two or more decay channels, all of which would have to be suppressed in order to prevent spontaneous emission. The transition observed is $(n=22,|m|=21) \rightarrow(n=21,|m|=20)$; the wave- length is $\lambda=0.45 \mathrm{~mm}$. The selection rule $\Delta|m|=-1$ assures that the radiation is polarized perpendicular to the quantization axis defined by an applied electric field.

In the usual mechanical treatment of spontaneous emission, the emission rate is proportional to the density of modes in free space. It is straightforward to show that in a cavity formed by infinite conducting plates separated by distance $d$, the mode density for the electric field parallel to the surface vanishes for $d<\lambda / 2$, and that for $d$ slightly greater than $\lambda / 2$ the emission rate at a distance $z$ from the midplane is ${ }^{7}$

$$
A^{\prime}=3 A_{0} \sin ^{2}(\pi z / d-\pi / 2),
$$

where $A_{0}$ is the Einstein $A$ coefficient for the transition. In our experiment the atoms sample all of the space between the plates, so that the sample-averaged decay rate is

$$
\begin{aligned}
& A^{\prime}=\frac{3}{2} A_{0} \text { for } \lambda / 2 d<1, \\
& A^{\prime}=0 \text { for } \lambda / 2 d>1 .
\end{aligned}
$$

If $\lambda / 2 d<1$ the emission rate is slightly enhanced relative to the free-space rate. As the plate spacing is reduced, spontaneous emission is abruptly inhibited when $\lambda$ becomes greater than $2 d$. (Quadrupolar radiation can occur on the transition $n \rightarrow n-2$, but its rate is negligible.) Alternatively, the spacing can be fixed and the wavelength varied, for instance by the Stark effect.

The experiment was performed with a thermal atomic beam of cesium. The beam passes sequentially through three regions: a production region where the atoms are transferred to the circular state, a drift region inside the parallel-plate cavity with a mean transit time approximately equal to the free-space radiation lifetime, and finally the detector where the arrival times of the $n=22$ atoms are recorded. Spontaneous emission changes the shape of the time-of-flight curve from the usual Maxwellian form for an atomic beam. ${ }^{8}$ When the emission is inhibited, the curve reverts to its usual form and there is a dramatic increase in the overall transmission. To switch from enhanced to in- 
hibited emission, an electric field is applied to change the wavelength a few percent by the Stark effect.

The ( $\left.n=22, m=2, n_{1}=2\right)$ state of cesium is populated by stepwise excitation with two pulsed dye lasers and the atoms are transferred to the circular state by use of a method described previously. ${ }^{6}$ Microwavefrequency multiphoton transitions are induced by adiabatic rapid passage in an electric field which decreases from 426 to $407 \mathrm{~V} / \mathrm{cm}$ in $10 \mu \mathrm{s}$. $30.7-\mathrm{GHz}$ radiation is used to drive the $|m|=2 \rightarrow 4$ transition; subsequent transitions are driven by $17.6-\mathrm{GHz}$ radiation. The production region consists of two $(5 \times 4)-\mathrm{cm}^{2}$ plates separated by $0.894-\mathrm{cm}$ spacers. The laser and microwave beams are perpendicular to the atomic beam and are polarized perpendicularly to the static electric field.

The cavity consists of two gold-plated aluminum plates, $6.4 \mathrm{~cm}$ wide $\times 12.7 \mathrm{~cm}$ long, that are separated by six disk-shaped quartz spacers. The spacer thickness, $230.1 \mu \mathrm{m}$, is $1.02\left(\lambda_{0} / 2\right)$, where $\lambda_{0}$ is the transition wavelength in zero field. The circular states have no first-order Stark shift but the transition wavelength $\lambda$ can be slightly increased via the second-order Stark effect. However, the maximum field that can be applied is limited by the onset of field ionization in the cavity. For the $n=22$ circular state the applied field must be less than $2600 \mathrm{~V} / \mathrm{cm}$. This allows a tuning range of $\Delta \lambda / \lambda_{0}=0.04$.

The atomic-beam detector consists of two $3.8-\mathrm{cm}$ square copper plates that are separated by approximately $0.5 \mathrm{~cm}$ and tilted from the parallel by an angle of $7^{\circ}$. The potential between the plates is maintained

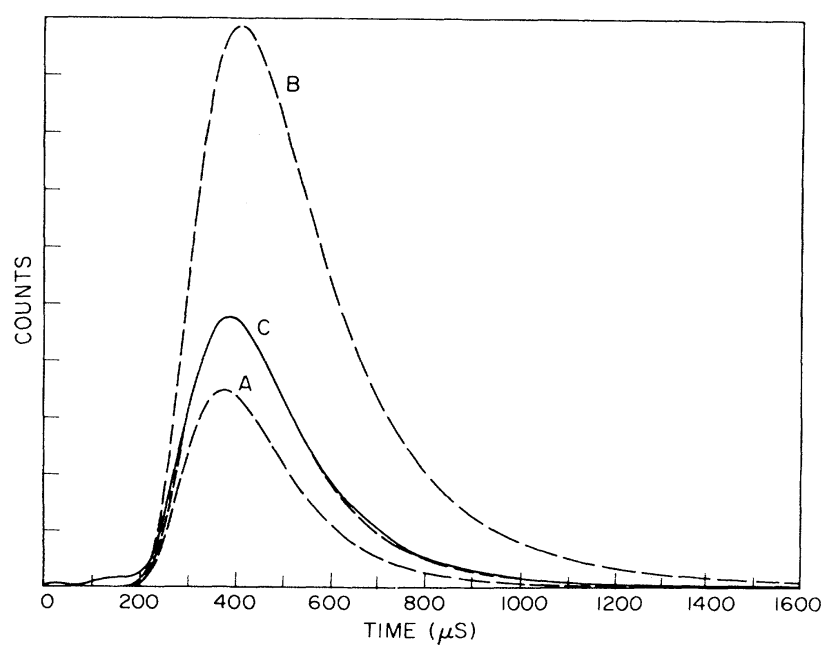

FIG. 1. Time-of-flight signals for various spontaneous decay rates. Mean time of flight $=1.5 / A_{0}$, where $A_{0}$ is the free-space radiative decay rate. Curve $A$, calculated signal, enhanced decay rate $A^{\prime}=\frac{3}{2} A_{0}$. Curve $B$, calculated signal, no radiative decay. Curve $C$, free space, $A^{\prime}=A_{0}$; calculated (dashed line), measured (solid line). at approximately $1100 \mathrm{~V}$. Because of the tilt, the atoms experience an increasing field as they pass between the plates. The ionization rates for the two states differ substantially, so that the location of a field-ionization event can serve to determine the atomic state. The initial state, $n=22$, ionizes approximately $0.7 \mathrm{~cm}$ into the detector, while the final state, $n=21$, ionizes approximately $0.5 \mathrm{~cm}$ further downstream. The combined effects of the large but finite increase of the ionization rate with field and the distribution of velocities of the atoms spreads the ionization signal over approximately $0.3 \mathrm{~cm}$. An aperture of this length in the negative-field plate allows the ions to pass to a charged-particle detector. The two states are effectively resolved. The signal from the chargedparticle detector passes to a multichannel scaler with a $2-\mu \mathrm{s}$ bin width.

In order to minimize blackbody effects the apparatus was operated at a temperature of $6.5 \mathrm{~K}$. The only effect of the $6.5-\mathrm{K}$ radiation was to reduce the free-space lifetime of the $n=22$ circular state from 460 to 451 $\mu \mathrm{s}$. To verify the accuracy of the time-of-flight measurement, the plate separation was increased to $15 \lambda_{0}$ and the free-space lifetime was measured. The timeof-flight distribution is given by

$$
\frac{d N}{d t}=\frac{N_{0}}{t_{0}}\left(\frac{t_{0}}{t}\right)^{5} e^{-\left(t_{0} / t\right)^{2}} e^{-\Gamma t}
$$

$\Gamma$ is the radiative decay rate. $t_{0}=L / \alpha$, where $L$ is the time-of-flight distance and $\alpha=(2 k T / M)^{1 / 2}$ is the most probable speed in the source. $N_{0}$ is a normalization

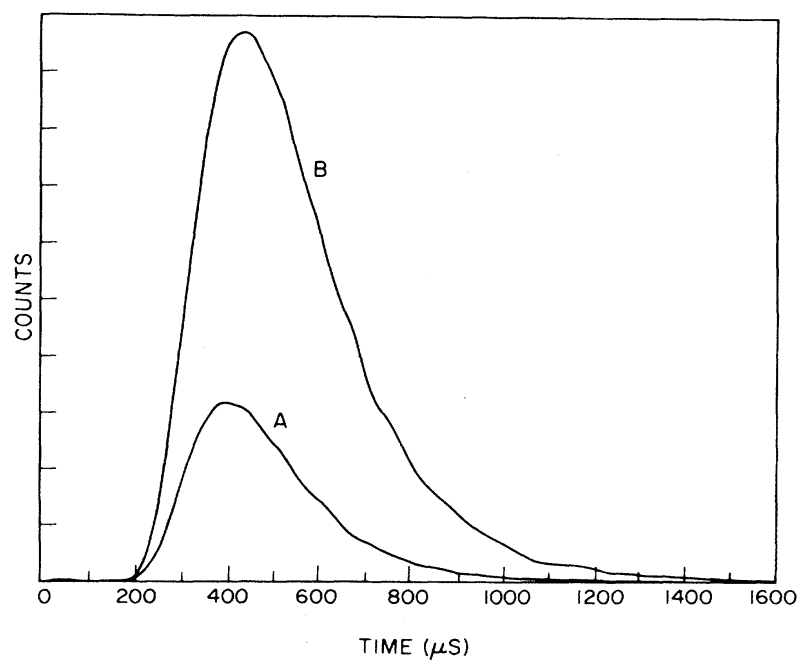

FIG. 2. Inhibited spontaneous emission. Time-of-flight data for inhibited spontaneous emission $(\lambda / 2 d>1$, curve $B)$ and enhanced spontaneous emission $(\lambda / 2 d<1$, curve $A)$ were taken simultaneously by modulation of the wavelength with an applied electric field. 
factor. Experimental data are compared with this distribution in Fig. 1, curve $C$. The measured lifetime in the $6.5-\mathrm{K}$ environment is $1 / \Gamma=450(10) \mu \mathrm{s}$, in excellent agreement with the calculated value, $451 \mu \mathrm{s}$. The principal sources of uncertainty are the oven temperature and the effects on $L$ of finite source and detector apertures.

To demonstrate inhibited spontaneous emission, the cavity plates were returned to their $\lambda / 2$ separation and time-of-flight data were accumulated in two channels as the electric field was alternated between a low value for which $\lambda / 2 d<1$ (enhanced emission) and a high value for which $\lambda / 2>d$ (inhibited emission). Typical results are shown in Fig. 2. The most striking feature is the increase in the total signal. This increase can serve to provide a measure of the altered decay rates, but accurate values of the rates must be obtained by
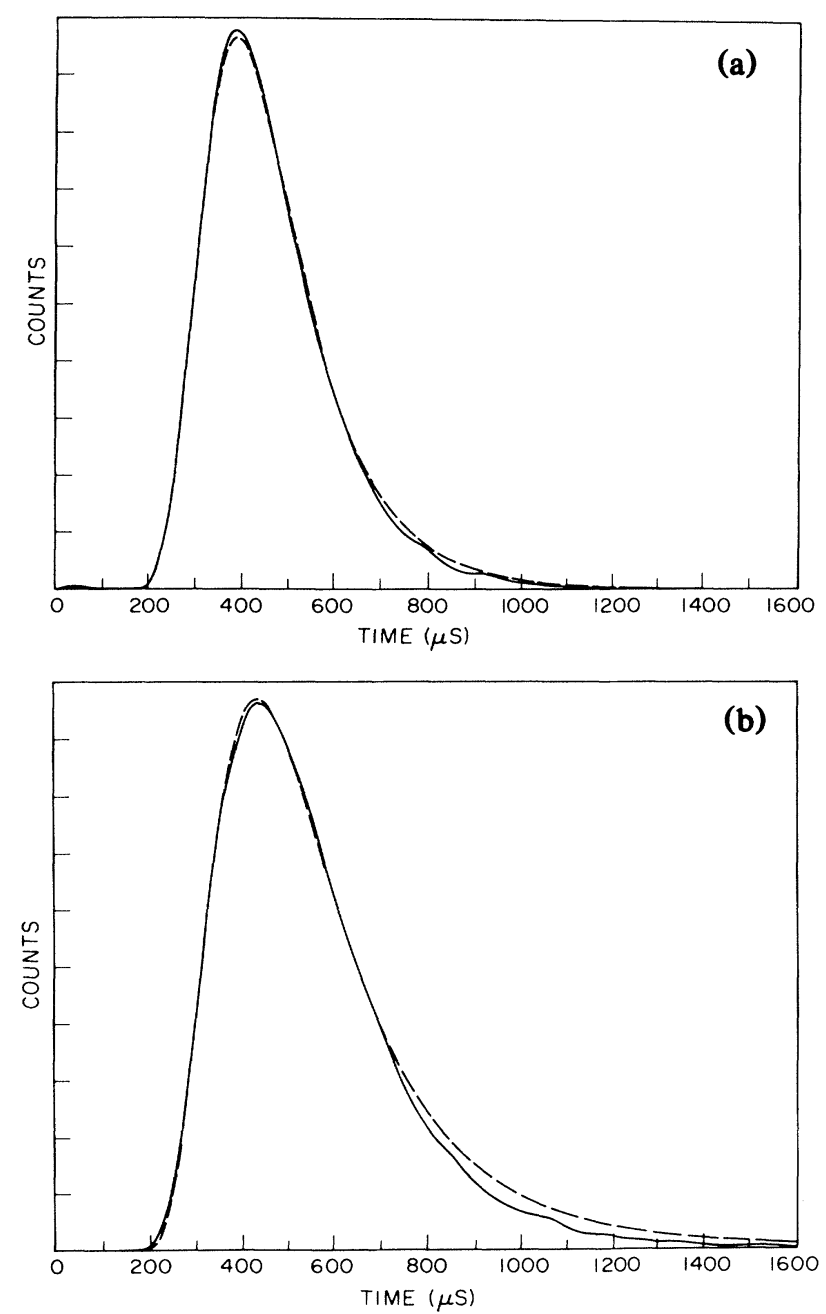

FIG. 3. (a) Experimental (solid) and theoretical (dashed) time-of-flight curves for enhanced spontaneous emission. (b) Same for inhibited spontaneous emission. curve fitting.

Figure 3 displays data and calculated time-of-flight curves for enhanced and inhibited emission. The decay rate is

$$
\Gamma=(l / L) A^{\prime}+(1-l / L) A_{0},
$$

where $l$ and $L$ are the cavity and total time-of-flight lengths, respectively; $l / L=0.79$. In plotting the calculated curve for enhanced emission, $A^{\prime}$ is taken to be the enhanced value just above cutoff: $\frac{3}{2} A_{0}$. In a curve-fitting analysis, the measured enhancement factor agrees with theory within its uncertainty of approximately $5 \%$. In plotting the calculated curve for inhibited emission, $A^{\prime}$ is taken to be zero. The curve fit in Fig. 3 is excellent for times less than $900 \mu \mathrm{s}$, but for longer times-corresponding to atoms with speeds less than $\frac{3}{4} \alpha$-there is a systematic depletion of the signal. Because the onset of the depletion is abrupt, the depletion cannot simply be a manifestation of a larger decay rate. We believe that the diminished number of slow atoms is due to a slight penetration by the fringe electric field of the cavity into the detector which causes a shift in the field-ionization location. Slow atoms ionize at a lower field than faster ones so that such an increase in field would cause them to ionize prior to reaching the detection aperture. Because of this systematic effect, data for times greater than 900 $\mu$ s were excluded in the final analysis, although their exclusion had little effect on the results.

In principle the time-of-flight data for inhibited emission could be fitted with a single parameter-the inhibited decay rate-with use of the enhanced-decay curves to provide normalization. However, the enhanced-decay data taken simultaneously with the usable inhibited-decay data were not good enough to provide a precise value for the normalization. Consequently, the inhibited-decay data were analyzed with two free parameters-normalization and decay rate-and the resulting errors analyzed by a chisquared test. The data were consistent with $A^{\prime}=0$ with an estimated limit of error of $\pm 0.05 A_{0}$. Thus the inhibited lifetime is estimated to be at least 20 times longer than the free-space lifetime.

The precise nature of the "turn on" of spontaneous emission is also of interest. This was measured by studying the total transmission as a function of $\lambda / 2 d$. Ideally, the turn on would be infinitely sharp at $\lambda / 2 d=1$. In reality, the transition is spread by the effective $Q$ of the cavity. Data for the total transmission are shown in Fig. 4. The width of the transition region corresponds to an effective $Q$ of approximately 120 . This can be accounted for chiefly by the lack of parallelism of the plates. Losses due to the finite conductivity of the plates and edge effects are negligible. The sharp drop in transmission for $\lambda / 2 d>1.015$ is due to the onset of field ionization in the drift region. A 


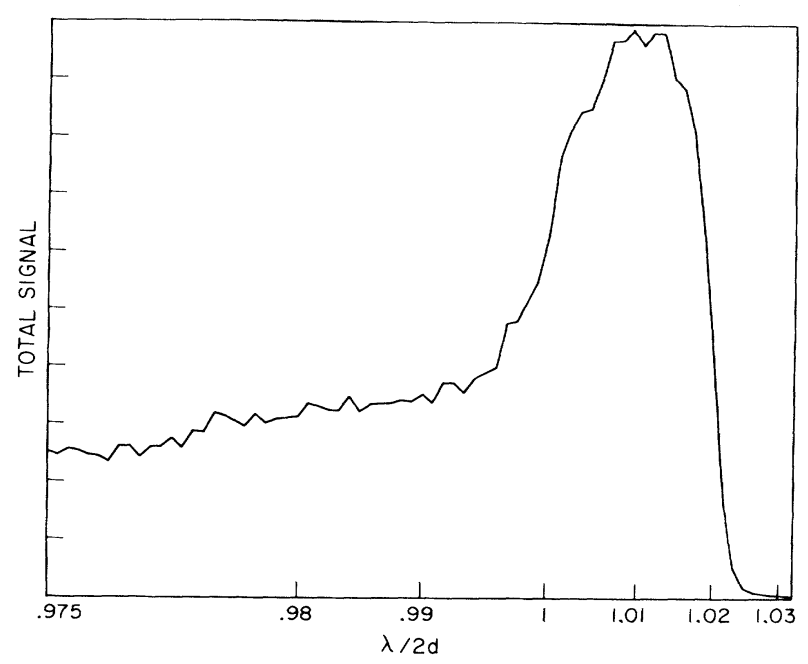

FIG. 4. Total transmission signal for $\lambda / 2 d$ near the cutoff region. ( $\lambda$ is altered by application of an electric field, which increases from 0 to $3.1 \mathrm{kV} / \mathrm{cm}$ for the data shown.) The sharp increase in transmission at $\lambda / 2 d=1$ is due to the inhibition of spontaneous emission; the decrease for $\lambda / 2 d$ $>1.015$ is due to field ionization between the cavity plates.

slight variation in transmission with $\lambda / 2 d$ is visible in the enhanced region. In this region the field between the field plates is increased from 0 to $1700 \mathrm{~V} \mathrm{~cm}^{-1}$. At low fields there is a small loss of atoms because of nonadiabatic effects that mix $m$ states as they pass from the production region into the cavity. As the field is increased this loss is eliminated.

Suppression of spontaneous emission offers the opportunity of eliminating the natural linewidth in spectroscopic measurements. However, in measuring energy levels to superhigh accuracy by elimination of spontaneous emission it must be remembered that the cavity inevitably introduces "nonradiative" shifts that alter the structure of the atom. ${ }^{1}$ In effect, the atomvacuum system is replaced by the atom-cavity system. There has been theoretical interest in such systems since the work of Casimir and Polder. ${ }^{9}$ The problem is now becoming experimentally important, for as Brown et al. ${ }^{10}$ have pointed out, cavity shifts are expected to be significant in forthcoming measurements of $g-2$ for a trapped electron. Furthermore, they must be taken into account in the design of future atomic clocks. Although cavity shifts may set the ultimate limit for useful applications of inhibited spontaneous emission as a high-precision technique, it should be pointed out that the present experiment provides a significant step toward very-high-precision spectroscopy of Rydberg atoms by achieving a major increase in their useful time-of-flight path length.

The authors thank Dr. Harald Hess for helpful advice on the cryostat design. This work was supported by the Joint Services Electronics Program under Grant No. DAAG29-83-K-0003, the National Science Foundation under Grant No. PHY84-11483, and the Office of Naval Research under Grant No. NOO14-79-C0183 .

\footnotetext{
(a) Present address: Time and Frequency Division, National Bureau of Standards, Boulder, Colo. 80303.

${ }^{1}$ D. Kleppner, in Atomic Physics and Astrophysics, edited by M. Chretien and E. Lipworth (Gordon and Breach, New York, 1971), p. 5.

${ }^{2}$ D. Kleppner, Phys. Rev. Lett. 47, 233 (1981).

${ }^{3}$ K. H. Drexhage, in Progress in Optics, edited by E. Wolf (North-Holland, Amsterdam, 1974), Vol. 12, p. 165.

${ }^{4}$ A. G. Vaidyanathan, W. P. Spencer, and D. Kleppner, Phys. Rev. Lett. 47, 1592 (1981).

${ }^{5}$ G. Gabrielse and H. Dehmelt, Phys. Rev. Lett. 55, 67 (1985).

${ }^{6}$ R. G. Hulet and D. Kleppner, Phys. Rev. Lett. 51, 1430 (1983).

${ }^{7}$ P. W. Milonni and P. L. Knight, Opt. Commun. 9, 119 (1973).

${ }^{8}$ T. Heindorff and B. Fischer, Rev. Sci. Instrum. 55, 347 (1984).

${ }^{9}$ H. B. G. Casimir and D. Polder, Phys. Rev. 73, 360 (1948). Some additional references are given in Ref. 4.

${ }^{10}$ L. S. Brown, G. Gabrielse, K. Helmerson, and J. Tan, Phys. Rev. Lett. 55, 44 (1985).
} 